\title{
Paternal alcohol exposure in mice alters brain NGF and BDNF and increases ethanol-elicited preference in male offspring
}

\author{
Mauro Ceccanti ${ }^{1 *}$, Roberto Coccurello ${ }^{2 *}$, Valentina Carito², Stefania Ciafrè ${ }^{3}$, \\ Giampiero Ferraguti ${ }^{4}$, Giacomo Giacovazzo ${ }^{2}$, Rosanna Mancinelli ${ }^{5}$, Paola Tirassa ${ }^{2}$, \\ George N. Chaldakov ${ }^{6}$, Esterina Pascale ${ }^{7}$, Marco Ceccanti ${ }^{8}$, Claudia Codazzo' \& Marco Fiore $^{2}$ \\ Centro Riferimento Alcologico Regione Lazio', Department of Cellular Biotechnologies and Hematology ${ }^{4}$, Department of Medical-Surgical Sciences and \\ Biotechnologies ${ }^{7}$, Department of Neurology and Psychiatry ${ }^{8}$, Sapienza University of Rome, Italy, Institute of Cell Biology and Neurobiology (IBCN)/IRCCS S. Lucia \\ Foundation, Italy ${ }^{2}$, Institute of Translational Pharmacology (IFT), National Research Council of Italy (C.N.R.), Italy ${ }^{3}$, Centro Nazionale Sostanze Chimiche, Instituto \\ Superiore di Sanità, Rome, Italy ${ }^{5}$ and Laboratory of Cell Biology, Medical University, Bulgaria ${ }^{6}$
}

\begin{abstract}
Ethanol (EtOH) exposure during pregnancy induces cognitive and physiological deficits in the offspring. However, the role of paternal alcohol exposure (PAE) on offspring EtOH sensitivity and neurotrophins has not received much attention. The present study examined whether PAE may disrupt nerve growth factor (NGF) and/or brain-derived neurotrophic factor (BDNF) and affect EtOH preference/rewarding properties in the male offspring. CD1 sire mice were chronically addicted for EtOH or administered with sucrose. Their male offsprings when adult were assessed for EtOH preference by a conditioned place preference paradigm. NGF and BDNF, their receptors ( $\mathrm{p} 75^{\mathrm{NTR}}$, TrkA and TrkB), dopamine active transporter (DAT), dopamine receptors D1 and D2, pro-NGF and pro-BDNF were also evaluated in brain areas. PAE affected NGF levels in frontal cortex, striatum, olfactory lobes, hippocampus and hypothalamus. BDNF alterations in frontal cortex, striatum and olfactory lobes were found. PAE induced a higher susceptibility to the EtOH rewarding effects mostly evident at the lower concentration $(0.5 \mathrm{~g} / \mathrm{kg})$ that was ineffective in non-PAE offsprings. Moreover, higher ethanol concentrations $(1.5 \mathrm{~g} / \mathrm{kg})$ produced an aversive response in PAE animals and a significant preference in non-PAE offspring. PAE affected also TrkA in the hippocampus and $\mathrm{p} 75^{\mathrm{NTR}}$ in the frontal cortex. DAT was affected in the olfactory lobes in PAE animals treated with $0.5 \mathrm{~g} / \mathrm{kg}$ of ethanol while no differences were found on D1/D2 receptors and for pro-NGF or pro-BDNF. In conclusion, this study shows that: PAE affects NGF and BDNF expression in the mouse brain; PAE may induce ethanol intake preference in the male offspring.
\end{abstract}

Keywords Ethanol intake preference, neurotrophin, paternal alcohol exposure.

Correspondence to: Marco Fiore, Institute of Cell Biology and Neurobiology (IBCN)-CNR, Via del Fosso di Fiorano 64, Roma 00143, Italy. E-mail: marco.fiore@cnr.it

\section{INTRODUCTION}

A great body of evidence suggests that maternal alcohol exposure in utero may be associated with a wide variety of anomalies in the offspring, such as pre- and postnatal growth retardation, a distinctive facial dysmorphology, damage to several organs, including heart, kidney and skeleton, and central nervous system dysfunction, resulting in cognitive deficits, hyperactivity, sleep disturbance, etc. (Jones 1975; Streissguth \& O'Malley 2000; O'Malley \&
Nanson 2002; Abel 2009). A clinically diagnosable pattern of these effects is termed fetal alcohol syndrome (FAS) that should not be confused with fetal alcohol spectrum disorders (FASDs), a condition that describes a continuum of permanent birth defects caused by maternal consumption of alcohol during pregnancy, which includes FAS (Astley 2004). Based also on the four-digit method of diagnosis (Astley 2013), there are also a number of other subtypes with evolving nomenclature and definitions settled on partial expressions of FAS,

*The authors contributed equally to this article. 
including partial fetal alcohol syndrome, alcohol-related neurodevelopmental disorder, alcohol-related birth defects, and fetal alcohol effect (Bakoyiannis et al. 2014).

Contrary to the great attention given to the influence that maternal factors have on the outcome of pregnancy, little is currently known about the possible role played by paternal factors, particularly about the influence of paternal alcohol exposure (PAE) on the neurobehavioral and developmental features of offspring (Abel 2004). It has been hypothesized that about 75 percent of children with FAS have heavy drinkers or alcoholic biological fathers (Abel 2004). These data suggest that the anomalies in the offspring attributed to the influence of teratogenic effects of maternal drinking are also the result of the PAE, so the anomalies may be due to or are exacerbated by paternal drinking. It has been demonstrated that offsprings sired by alcohol-exposed fathers have some difficulty in certain learning tasks, e.g. passive avoidance (Abel \& Dintcheff 1986). Recent studies demonstrated also that PAE can increase the percentage of fetuses with the human equivalent of low birth weight (Abel 2004). Other studies established that animals sired by alcohol-treated fathers showed an increase in major malformations or average fetal or birth weight (Randall et al. 1982; Bielawski \& Abel 1997; Abel 1999). It has been also shown that paternal chronic vapor ethanol reduced ethanol preference and consumption, enhanced sensitivity to the anxiolytic and motor-enhancing effects of ethanol compared with control-sired male offspring (Finegersh \& Homanics 2014). Unlike the effects observed as a result of maternal alcohol exposure, which are probably due to the direct effect and disruption of normal morphogenesis in the developing embryo and fetus (Goodlett et al. 1989; Goodlett \& Horn 2001), the effects observed after PAE are difficult to explain. In this regard, three possible mechanisms have been proposed involving non-genetic, genetic and epigenetic factors (Abel 2004, 2009).

As for maternal ethanol exposure, important effects have been shown in brain regions inducing neuronal cell death in the offspring (Servais et al. 2007), with significant changes at the neurotrophin signaling pathways level (Aloe \& Tirassa 1992; Aloe, Bracci-Laudiero \& Tirassa 1993; Aloe 2006; Fiore et al. 2009b,c; Ceccanti et al. 2012, 2013). Similar to what occurs as a result of maternal prenatal alcohol exposure, paternal alcohol consumption could affect biological mediators, such as neurotrophins, molecules known to play a crucial role in the survival, development and function of nervous system (Sofroniew, Howe \& Mobley 2001; Chao, Rajagopal \& Lee 2006). Among these, nerve growth factor (NGF) and brain-derived neurotrophic factor (BDNF) are prominent growth factors that play a critical role in the physiopathology of the brain and cardiometabolic system (Chao 2000; Fiore, Chaldakov \& Aloe 2009a; Yanev et al. 2013). NGF was the first neurotrophin discovered, about 60 years ago by Rita Levi-Montalcini and colleagues, as a soluble protein that promotes the survival and growth of sympathetic and sensory neurons during development (Cohen, Levi-Montalcini \& Hamburger 1954; Levi-Montalcini 1987). Several data then showed that NGF exerts trophic effects on NGF-responsive neurons, not only during the development but also in adulthood (Alleva, Aloe \& Bigi 1993; Aloe 2001; Aloe, Alleva \& Fiore 2002). Despite the NGF action was once considered limited to central and peripheral nervous systems, more studies have widely demonstrated that NGF actions also extended to several non-neuronal cells. In this regard, NGF can affect the functioning of the immune-hematopoietic system and it plays an important role in the maintenance of a balance between neuroendocrine, immune and metabolic systems (Aloe et al. 1997; Bonini et al. 2002; Fiore et al. 2009a; Yanev et al. 2013). Furthermore, BDNF is a growth factor expressed in a range of tissue and cell types, such as the central and peripheral nervous system, retina and adipose tissue (Allen \& Dawbarn 2006; Chao et al. 2006; Yanev et al. 2013). BDNF plays an important role in dendritic branching and dendritic spine plasticity associated with learning and memory processes (He et al. 2013; Bekinschtein, Cammarota \& Medina 2014). There is also evidence showing that the precursors to NGF or BDNF, pro-NGF and pro-BDNF, may also play important roles due to their 'mixed' apoptotic and trophic properties (Hempstead 2014). Both NGF and BDNF are widely known to play an important role within the pathogenesis of developmental alcohol exposure since it has been shown that alcohol exposure during pregnancy disrupts neurotrophin pathways that in turn affect brain cell growth and development (Aloe 2006; Fiore et al. 2009b) with or without the contribution of other mechanisms leading to FASD onset: i.e. the FASD glutamatergic hypothesis (Tsai, Gastfriend \& Coyle 1995).

The biological effects of these two neurotrophins are mediated through activation of the members of the tropomyosin-related kinase (Trk) family of receptor tyrosine kinases: NGF activates TrkA, BDNF bind to TrkB (Freund-Michel \& Frossard 2008). Both NGF and BDNF are able to activate the low affinity p75 neurotrophin receptor (p75 ${ }^{\mathrm{NTR}}$ ) (Chao \& Hempstead 1995; Huang \& Reichardt 2003).

Thus, the aim of the present work was to investigate in the mouse the effects of PAE on the NGF and BDNF levels in the offspring brain and on the expression of their receptors, TrkA, TrkB and p75 $5^{\mathrm{NTR}}$. In our study, we assessed PAE offspring as EtOH-induced preference to determine whether or not paternal chronic alcohol exposure affects the postnatal experiences on processing of rewarding and aversive stimuli. We also investigated the expression of pro-NGF, pro-BDNF and the expression of 
the dopamine active transporter (DAT) and dopamine receptors D1 and D2 known to have a subtle role in alcohol dependence and associated rewarding mechanisms (Tabakoff \& Hoffman 2013).

\section{MATERIALS AND METHODS}

\section{Animals}

CD1 outbred male mice were used in this experiment. All animals were 3 months old and housed singularly at the beginning of the experiments in Plexiglas cages $(33 \times 13 \times 14 \mathrm{~cm})$ under standardized conditions with pellet food (enriched standard diet purchased from Mucedola, Settimo Milanese, Italy). Food (Purina Lab Chow \#5015) and water were available ad libitum. A 12L:12D lighting regime was used.

Ten male CD1 mice were randomly divided into two groups: a group of mice $(n=5)$ received ad libitum, as only source of liquid, after an habituation period of 10 days, ethanol 11 percent dissolved in water for 60 days; another group of mice $(n=5)$ received sucrose dissolved in water at equivalent caloric intake of the ethanol group and was used as control group. Ethanol used for the preparation of the drinking solution was obtained from Sigma-Aldrich (St. Louis, MO, USA) and was of analytical grade. The daily consumption of ethanol solution was measured day by day. Two months after treatment, males were allowed to breed with non-treated females $(n=10)$, for 2 hours, without any source of liquid. The presence of a copulation plug in female mice was presumed to indicate mating. At birth, all litters were reduced to four males only (Cirulli, Adriani \& Laviola 1997). Pups remained with their own mother. The experiments were carried out only on the male offspring (20 offsprings sired by control fathers and 20 offsprings sired by alcohol-exposed fathers). To investigate ethanol consumption preference in the offspring, 60-day-old offsprings were treated i.p. with 0.5 or $1.5 \mathrm{~g} / \mathrm{kg}$ of ethanol or vehicle (saline solution) to generate six experimental groups, namely: PAE-Veh; PAE-0.5 EtOH; PAE-1.5 EtOH; non-PAE-Veh; non-PAE-0.5 EtOH; nonPAE-1.5 EtOH ( $n=6$ for each experimental group). All efforts were made to minimize and reduce animal suffering and for limiting the number of animals used. All animal experiments were carried out following the procedure described in the guidelines of the European Community Council Directive of 24 November 1986 (86/609/EEC).

\section{Place conditioning}

The procedure for evaluating EtOH-induced conditioned place preference (CPP) has been described previously (Ventura et al. 2013). The place conditioning apparatus comprised two Plexiglas chambers $(15 \times 15 \times 20 \mathrm{~cm})$, one white with a large grid floor and the other black with a narrow grid floor and a central alley $(15 \times 5 \times 20 \mathrm{~cm})$. Two sliding doors $(4 \times 20 \mathrm{~cm})$ connected the alley to the chambers. In each chamber, two triangular parallelepipeds $(5 \times 5 \times 20 \mathrm{~cm})$ made of black Plexiglas and arranged in different patterns (always covering the surface of the chamber) were used as conditioned stimuli. The luminance of the chambers was adjusted so that visual and tactile cues would not induce unbiased preferences for a specific chamber. Briefly, on day 1 (preconditioning), PAE or non-PAE mice were placed between the two chambers free to explore the entire apparatus for 20 minutes. During the following 8 days (conditioning phase), each mouse was confined daily for 40 minutes alternately in one of the two chambers. During EtOH conditioning, animals were randomly assigned to receive EtOH $(0,0.5$ or $1.5 \mathrm{~g} / \mathrm{kg}$, prepared from 20 percent ethanol solution in isotonic saline, v/v). For all three groups $(0,0.5$ and $1.5 \mathrm{~g} / \mathrm{kg})$, pairings were counterbalanced so that for half of each group the unconditioned stimulus (EtOH) was paired with one of the patterns and for half with the other (saline). Testing for the expression of CPP was conducted on day 10 (post-conditioning) using the pretest procedure during which the total time spent in each chamber was recorded. Behavioral data were collected and analyzed by the 'Etho-Vision' (Noldus, the Netherlands) fully automated video tracking system. The experimental system is recorded by a digital video camera and the signal digitized (by a hardware device called 'frame grabber') and transmitted to computer's data storage. Next, the digital data were analyzed by means of the Etho-Vision software to obtain the 'time spent' (seconds) that was used for each subject as raw data for preference scores in each sector of the apparatus. CPP was determined by plotting the time spent in the EtOH-paired compartment during the post-conditioning phase (day 10).

\section{Blood and brain tissue dissection}

Animals were sacrificed by a guillotine 7 days after the end of the behavioral experiments. The blood was collected in heparin vials and quickly centrifuged at $10000 \mathrm{rpm}$ for 15 minutes. The brain was quickly removed and tissues dissected out using a mouse brain matrix (ASI Instruments, Inc. Co., Warren, MI, USA) (Cuello 1983) and stored at $-80^{\circ} \mathrm{C}$ until used. Brain tissues were then homogenized and centrifuged at $8500 \mathrm{rpm}$ and the supernatant used for NGF and BDNF determination and Western blot analysis.

Blood ethanol levels by gas chromatography/head space (HS) procedure

Gas chromatography/HS is applied in this research to determine blood alcohol concentration in whole blood 
samples. In this research, we used a Clarus 600 Gas Chromatography Perkin Elmer and a TurboMatrix 40 Trap HeadSpace Sampler Perkin Elmer with FID detector. Analytical conditions were set up and the method was validated by a previous study (Macchia et al. 1995). From each blood sample is collected $100 \mu \mathrm{l}$ of whole blood with a micropipette and transferred this volume into a gas chromatography (GC) vial. The gasses that are formed inside GC vial will be collected in GC to be analyzed. If the sample is not analyzed in the same day as its collection, it is important to firmly close the vial to prevent the evaporation of ethanol during time and to conserve the vial inside of a refrigerator. Standard solutions were set up for calibration curve at 100, 50, 25, 12.5, 6.2 and $3.1 \mathrm{mg} \%$ of pure ethanol and were obtained by consequent dilutions of pure ethanol in distilled water.

\section{NGF and BDNF determination}

NGF and BDNF were measured following indications previously released (De Nicolo et al. 2013) in the hippocampus, frontal cortex, olfactory lobes, hypothalamus, liver and kidney $(n=4)$. NGF/BDNF evaluation was carried out with ELISA kits 'NGF EmaxTM ImmunoAssay System number G7631' and 'BDNF EmaxTM ImmunoAssay System number G7611' by Promega (Madison, WI, USA) following the instructions provided by the manufacturer. The NGF sensitivity of the assay was about $3 \mathrm{pg} / \mathrm{g}$ of wet tissue and cross-reactivity with other related neurotrophic factor (BDNF, neurotrophin-3 and neurotrophin-4) was less than 3 percent. The BDNF sensitivity of the assay was about $15 \mathrm{pg} / \mathrm{ml}$ of wet tissue and cross-reactivity with other related neurotrophic factor (NGF, neurotrophin-3 and neurotrophin-4) was less than 3 percent. Data are represented as $\mathrm{pg} / \mathrm{mg}$ total proteins and all assays were performed in duplicate which were averaged for statistical comparison.

Western blotting analyses for TrkA, TrkB, p7 ${ }^{\mathrm{NTR}}$, DAT, D1, D2, pro-NGF and pro-BDNF

TrkA, TrkB, p75 ${ }^{\text {NTR }}$, DAT, D1, D2, pro-NGF and pro-BDNF were measured in the hippocampus, hypothalamus, olfactory lobes and frontal cortex $(n=4)$ following methods previously described (De Nicolo et al. 2013). Aliquots $(30 \mathrm{mg})$ from total tissue lysate proteins were resolved on 10 percent SDS-PAGE gels and analyzed by immunoblotting with the following antibodies: anti-TrkA 1:1000 (provided by Santa Cruz, CA, USA, catalog number: 763:sc-118), anti-TrkB 1:1000 (provided by BD Biosciences Pharmingen, San Jose, CA, USA, catalog number: 610102), anti-p75NTR 1:1000 (provided by Cell Signaling, Danvers, MA, USA, catalog number: 8238), anti-DAT (provided by Millipore, Billerica, MA, USA, catalog number: AB1591P), anti-D1 1:1000 (provided by Abcam, Cambridge, UK, catalog number: AB20066), anti-D2 1:1000 (provided by Merck Millipore, Billerica, MA, USA, catalog number: AB5084P), anti-proNGF 1:1000 (provided by Alomone Labs, Israel, catalog number: ANT-005) or anti pro-BDNF 1:2000 (provided by Millipore, catalog number: AB5613P). The secondary antibodies used were as follows: horseradish peroxidaseconjugated anti-rabbit IgG or anti-mouse IgG (Cell Signalling, Beverly, MA, USA). The blots were developed with enhanced chemiluminescence assay (Amersham Bioscience, Amersham, UK), following the instructions of the manufacturer.

\section{Statistical analysis}

Data were analyzed by two-way ANOVAs considering as factors the paternal ethanol exposure and the ethanol treatment. Post hoc comparisons were performed using the Bonferroni's test.

\section{RESULTS}

\section{General description of the PAE mouse model}

Ethanol drinking did not elicit changes in the body weight of fathers, indeed at the time of mating there were not significant differences in body weight between the ethanol group of sires and the sucrose group $(49.33 \pm 2.21$ versus $47.97 \pm 1.33$, respectively). Liquid and food consumption of ethanol-exposed fathers and control fathers were also similar between groups. In the ethanol group of fathers, a careful day-by-day measurement of liquid consumption indicated that values of ethanol intake are comprised of 0.15 and $0.26 \mathrm{ml}$ with a mean daily consumption of $0.20 \pm 0.018 \mathrm{ml}$. The blood ethanol levels in the ethanolexposed sires 1 day after mating, expressed as $\mathrm{mg} / 100 \mathrm{ml}$ of mouse blood, were comprised of 3.5 and $21.5 \mathrm{mg} /$ $100 \mathrm{ml}$. As for the PAE offsprings, no differences in perinatal mortality, numbers of pups (litter size) and numbers of dead-born pups were observed. Quite interestingly, PAE offsprings had significant lower values of body weight as shown at the time of mouse sacrifice when compared with the body weight values of controls offspring without any effects of ethanol treatment $(42.88 \pm 0.65$ versus $47.24 \pm 0.69 \mathrm{~g} ; P<0.05$ in the ANOVA for the effect of ethanol administration).

\section{NGF determination}

Figure 1 shows the results of the ELISA for NGF (expressed as pg/mg total protein) in the offspring sired by alcohol-treated fathers or sucrose control fathers. In the cortex, data show lower NGF levels in PAE-0.5 EtOH but highest levels in non-PAE-Vehicle $(P s<0.05$ for the 
NGF
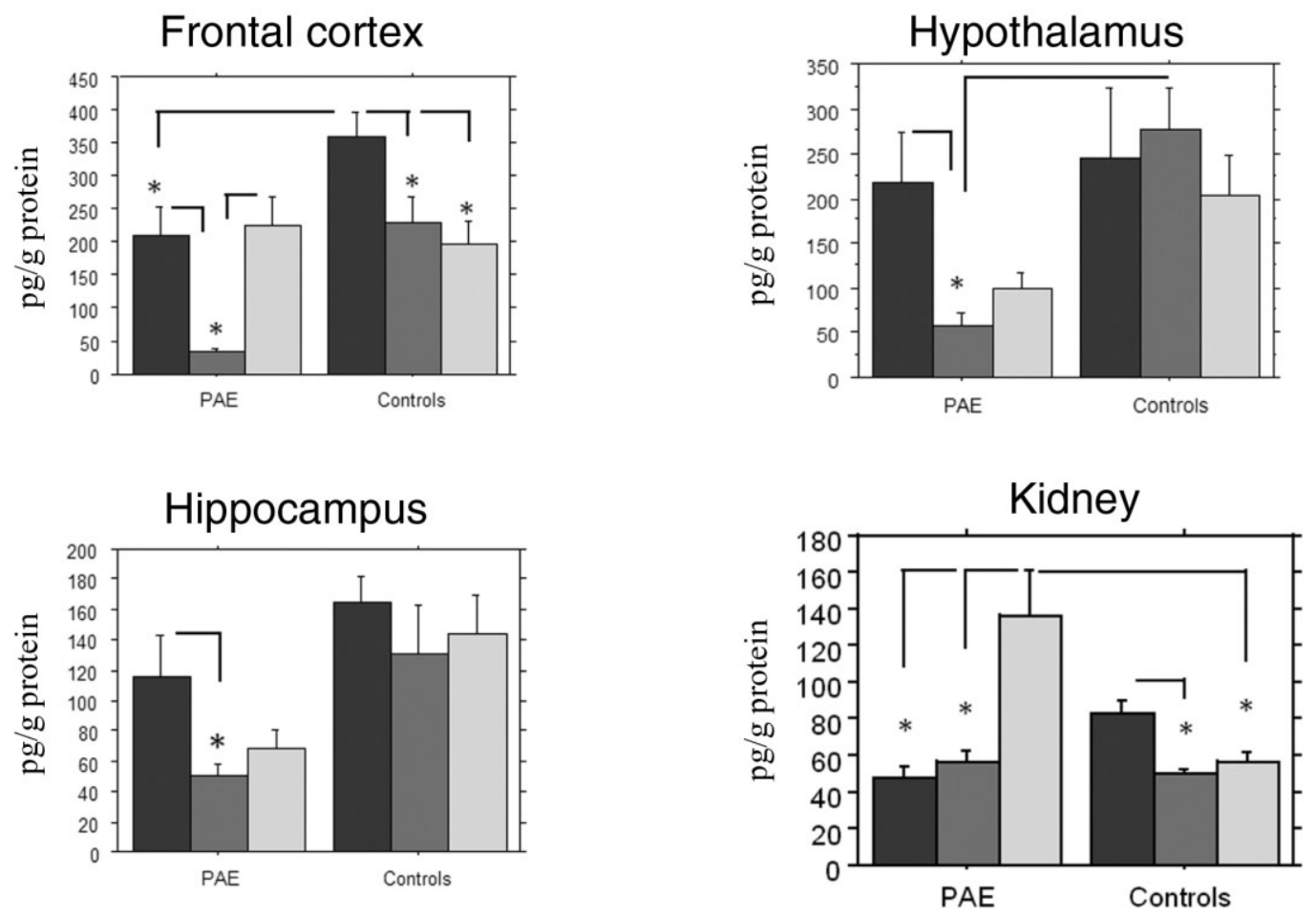

\section{Olfactory lobes}

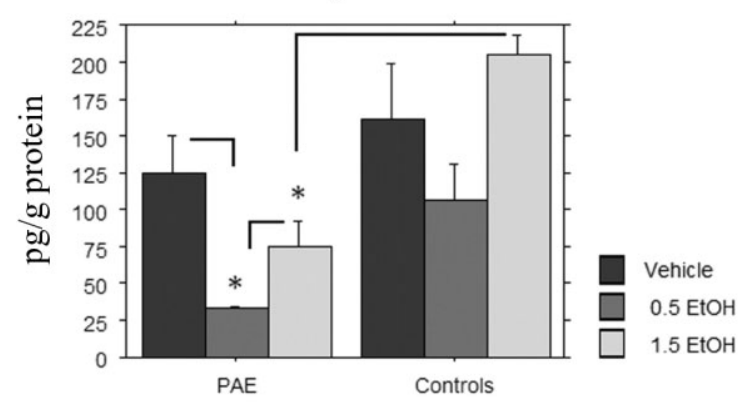

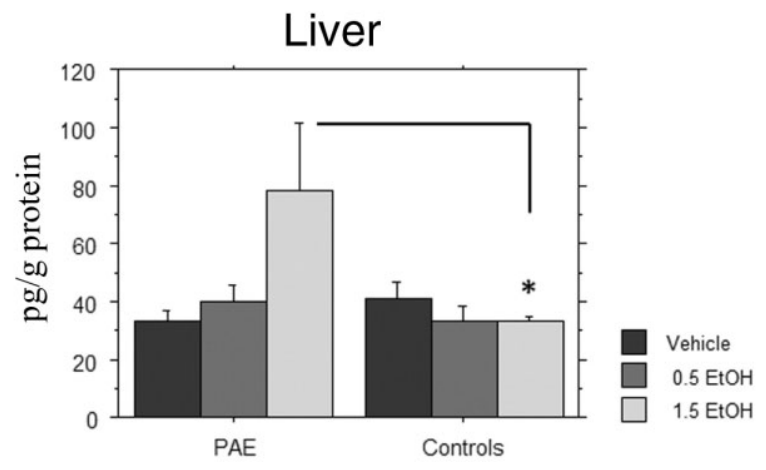

Figure I NGF levels expressed as pg/g proteins in selected areas (frontal cortex, hippocampus, olfactory lobes hypothalamus, kidney and liver) of male mice sired by control fathers or alcohol-exposed fathers and treated i.p. with 0.5 or $1.5 \mathrm{~g} / \mathrm{kg}$ of ethanol or vehicle (PAE-Veh; PAE-0.5 EtOH; PAE-I.5 EtOH; controls-Veh; controls- $0.5 \mathrm{EtOH}$; controls- I.5 EtOH). The vertical lines in the figure indicate pooled standard error means (SEM) derived from appropriate error mean square in the ANOVA. Asterisks indicate significant differences between groups $(* P<0.05)$

effects of PAE, ethanol treatment and PAE $\times$ ethanol treatment interaction; see post hocs for differences between groups). In the hippocampus, ANOVA revealed the effect of PAE due to the low NGF levels in PAE-0.5 EtOH mice $(P<0.01$ for the PAE effect, $P<0.05$ in post hocs). In the olfactory lobes, data showed effects of both PAE and ethanol administration $(P s<0.01$ for the effects of PAE and ethanol treatment; see post hocs for differences between groups). In the hypothalamus, ANOVA revealed the effect of PAE due to the low NGF levels in both PAE-0.5 EtOH and PAE-1.5 EtOH mice $(P<0.01$ for the PAE effect,
$P<0.05$ in post hocs). Quite interestingly, in the liver NGF levels were higher in PAE-1.5 EtOH animals when compared with the respective non-PAE $(P<0.05$ for the interaction PAE $\times$ ethanol treatment; see post hoc for differences between groups). Similar results were found in the kidney with a NGF potentiation in PAE-1.5 EtOH mice compared with their non-PAE $(P s<0.01$ for the effects of ethanol treatment and PAE $\times$ ethanol treatment interaction; see post hocs for differences between groups). No significant differences between groups were observed in the testis (data not shown). 


\section{BDNF}

\section{Frontal cortex}
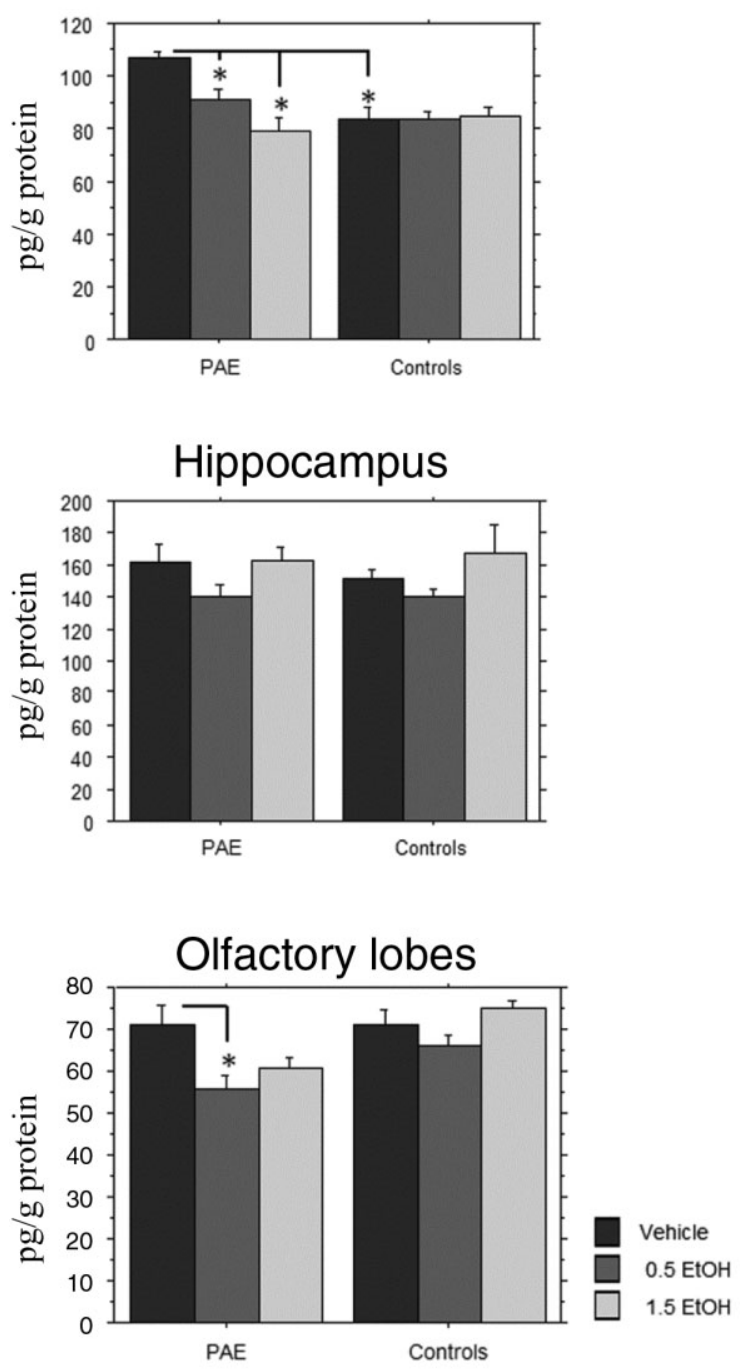
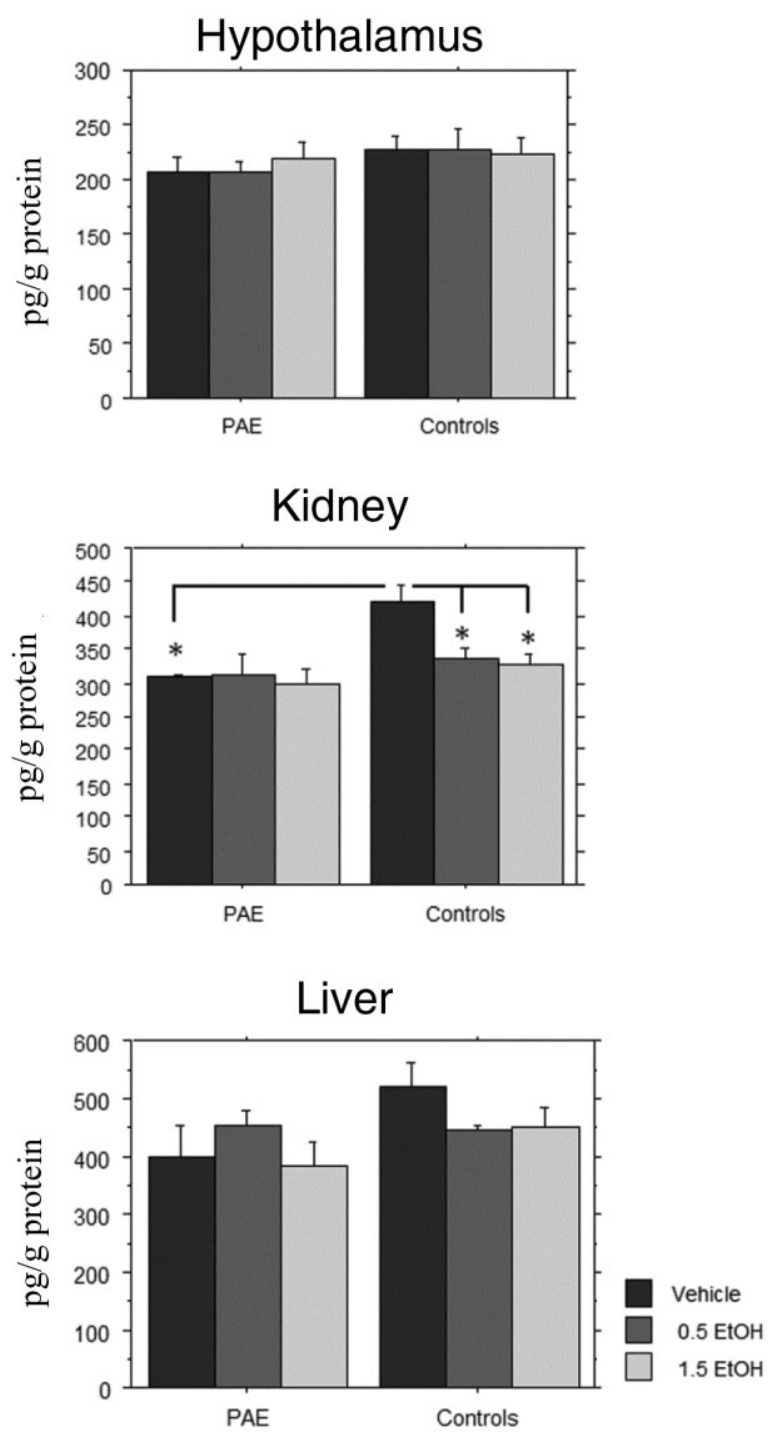

Figure 2 BDNF levels expressed as pg/g proteins in selected areas (frontal cortex, hippocampus, olfactory lobes hypothalamus, kidney and liver) of male mice sired by control fathers or alcohol-exposed fathers and treated i.p. with 0.5 or $1.5 \mathrm{~g} / \mathrm{kg}$ of ethanol or vehicle (PAE-Veh; PAE-0.5 EtOH; PAE-I.5 EtOH; controls-Veh; controls-0.5 EtOH; controls- I.5 EtOH). The vertical lines in the figure indicate pooled standard error means (SEM) derived from appropriate error mean square in the ANOVA. Asterisks indicate significant differences between groups $(* P<0.05)$

\section{BDNF determination}

Figure 2 shows the results of the ELISA for BDNF (expressed as $\mathrm{pg} / \mathrm{mg}$ total protein) in the offsprings sired by alcohol-treated fathers or sucrose control fathers. In the cortex, data show higher BDNF levels in PAE-Veh compared with the other groups (Ps $<0.05$ for the effects of PAE, ethanol treatment and PAE $\times$ ethanol treatment interaction; see post hocs for differences between groups). In the olfactory lobes, ANOVA revealed a modulation due to ethanol administration in PAE animals with higher concentrations in PAE-Veh compared with the other
PAE groups $(P s<0.01$ for the effects of both PAE and ethanol administration; $P<0.05$ in post hocs). In the hippocampus, data showed an effect of ethanol administration with low levels in the 0.5 groups $(P<0.05$ for the effects of ethanol treatment). In the hypothalamus, no differences between groups were found. In the liver, ANOVA just failed full significance for a BDNF decrease due to PAE ( $P=0.0569$ for the effect of PAE). In the kidney, a BDNF potentiation was found in non-PAE-Veh animals compared with the other groups $(P s<0.05$ for the effects of both PAE and ethanol treatment; see post hocs for differences between groups). No significant 
differences between groups were observed in the testis (data not shown).

Western blotting analyses for TrkA, TrkB, p7 $5^{\mathrm{NTR}}$, DAT, D1, D2, pro-NGF and pro-BDNF

Protein tissue extracts have been used to analyze (by Western blot) the expression of NGF and BDNF receptors (TrkA, TrkB and p75 ${ }^{\mathrm{NTR}}$ ), pro-NGF/pro-BDNF, dopamine receptors (D1 and D2) and the dopamine transporter (DAT) in different brain areas, such as: hippocampus, hypothalamus, frontal cortex and olfactory lobes. The data obtained by densitometric analysis in the offspring sired by alcohol-treated fathers or sucrose control fathers showed no significant differences in the protein expression of NGF and BDNF receptors, except for TrkA in the hippocampus and $\mathrm{p} 75^{\mathrm{NTR}}$ in the frontal cortex (Fig. 3). In the hippocampus (Fig. 3a), data showed higher TrkA expression in all PAE groups compared with respective non-PAE groups $(P<0.05$ for the effects of PAE in the ANOVA, see post hocs in the table for differences between groups). In the frontal cortex (Fig. 3b), data evidenced low p7 $5^{\mathrm{NTR}}$ expression in non-PAE-1.5 EtOH, in PAE-Veh and in PAE-0.5 EtOH when compared with non-PAE-Veh $(P=0.01$ in the ANOVA for the interaction PAE $\times$ Ethanol administration; see post hocs comparison in the table for the differences between groups). As for the expression of DAT (Fig. 3c), ANOVA revealed that the administration of 0.5 of ethanol elicited a strong DAT potentiation in the olfactory lobes $(P<0.01$ for the effect of ethanol administration; see post hocs in the table for differences between groups). No differences were found in the protein expression of $\mathrm{D} 1$ and $\mathrm{D} 2$ receptors in brain tissues or for pro-NGF and pro-BDNF (data not shown).

\section{Place conditioning}

The effects of EtOH-elicited preference in PAE or non-PAE male offsprings are shown in Fig. 4a and b, respectively. There was no statistically significant difference in preference for one chamber of the apparatus in control or EtOH groups during the pretest session (data not shown). For post-conditioning session in PAE offspring, the repeated measures ANOVA evidenced a significant $\mathrm{EtOH} \times$ pairing interaction $(P<0.01)$. Post hoc analysis revealed the existence of a significant preference for the EtOH-paired compartment only in mice conditioned with the lower dose of EtOH (0.5 mg/kg; Fig. 4a), while mice conditioned to the higher dose of EtOH $(1.5 \mathrm{mg} / \mathrm{kg})$ showed a significant increase in preference toward the EtOH unpaired (Fig. 4a) and therefore no preference when the compartment was paired to the higher dose tested. For post-conditioning session in non-PAE offspring, the repeated measure ANOVA showed a significant $\mathrm{EtOH} \times$ pairing interaction
$(P<0.001)$. Post hoc analysis further revealed a significant preference for the EtOH-paired compartment only in the non-PAE offspring conditioned with the higher dose of EtOH (1.5 mg/kg; Fig. 4b), while the offspring conditioned to the lower dose of EtOH $(0.5 \mathrm{mg} / \mathrm{kg})$ showed no difference as compared with the control group (Fig. 4b). Finally, there were no significant differences in locomotion between control and EtOH-conditioned mice during the conditioning phase (data not shown).

\section{DISCUSSION}

This is the first study to demonstrate that PAE affects NGF (Fig. 1) and BDNF (Fig. 2) levels in targeted brain regions and peripheral tissues from mice offspring and enhances the sensitivity to the rewarding effects of EtOH administration in male progeny. Our data provide evidence that EtOH-sired male offspring displayed a CPP to an environment paired with a non-reinforcing dose of EtOH (0.5 g/ $\mathrm{kg}$ ). The PAE-induced lowering of reward threshold to EtOH effects in mice offspring is further underlined by the aversive response induced by the higher $\mathrm{EtOH}$ dose $(1.5 \mathrm{~g} / \mathrm{kg})$. Thus, the EtOH dose eliciting a maximal place conditioning effect in the offspring of non-PAE mice produced a conditioned place aversion in the offspring of PAE mice, while a subthreshold dosage in non-PAE mice offspring produced a rewarding effect in male offspring of alcoholic fathers.

Our study provides evidence that PAE may affect the levels of key trophic factors as NGF and BDNF in the offspring brain, affecting also TrkA in the hippocampus and p75NTR in the frontal cortex. Our data show that NGF is a neurotrophin particularly vulnerable to alcoholinduced transgenerational neuronal damage. We found a marked decrease in NGF expression in the frontal cortex of male offspring from PAE mice, as compared with offspring from non-PAE animals, regardless of the dose of EtOH administrated (Fig. 1a). However, NGF levels also showed higher susceptibility to EtOH administration at hippocampal, hypothalamic and olfactory lobe levels (Fig. 1b, c and e). Indeed, 8 days of EtOH administration was sufficient to reduce the NGF expression in these brain areas below the level showed by the untreated offspring from PAE mice. Notably, the lower the dose of EtOH administered, the greater the reduction of NGF expression detected. In this regard, the increased sensitivity to rewarding effects of EtOH administration appears inversely related to the levels of NGF expressed in the frontal cortex, hippocampus, hypothalamus and olfactory lobes. By contrast, BDNF levels were increased only in the frontal cortex of PAE offspring that did not receive any EtOH treatment. Higher levels of BDNF mRNA expression have been detected in the amygdala and ventral tegmental area of alcohol preferring animals 
(a)

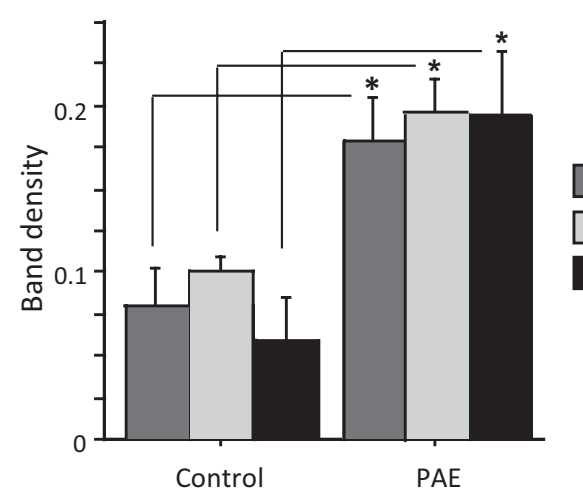

TrkA hippocampus

(b)
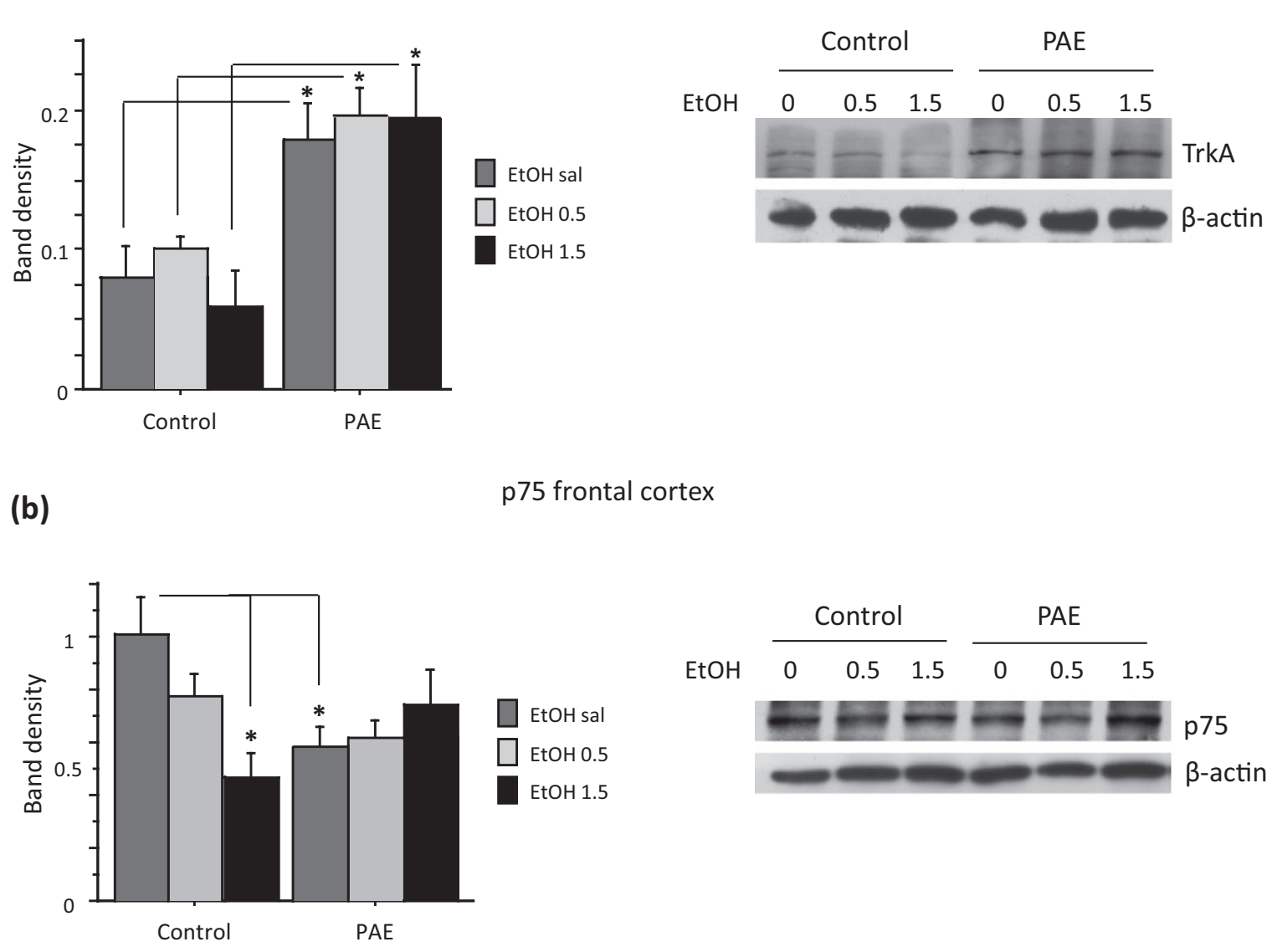

p75 frontal cortex

(c)

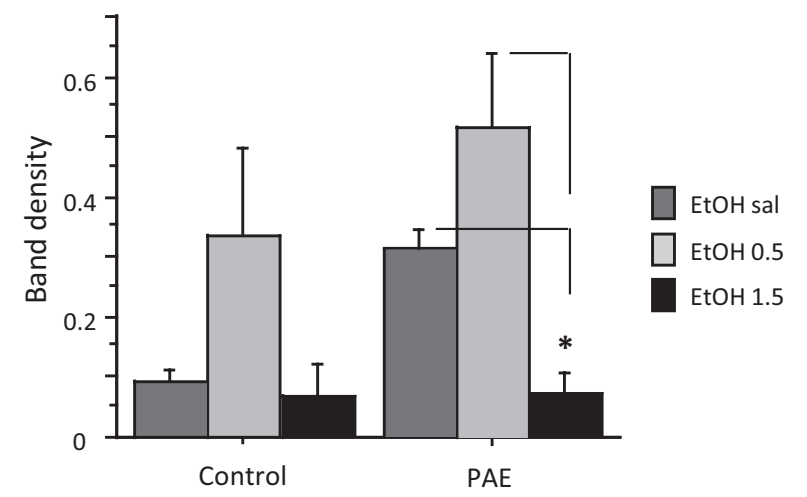

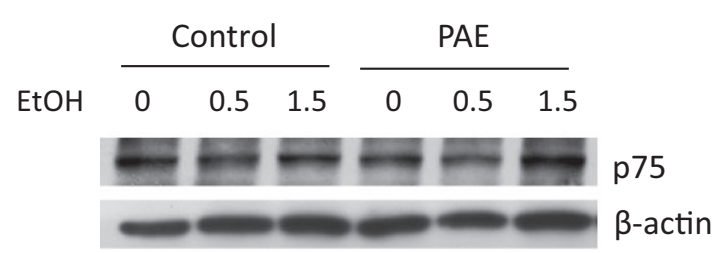

Figure 3 Densitometric analysis and gel expression of TrkA in the hippocampus (a), p $75^{\mathrm{NTR}}$ in the frontal cortex (b), and DAT in the olfactory lobes (c) of male mice sired by control fathers or alcohol-exposed fathers and treated i.p. with $0.5 \mathrm{or} 1.5 \mathrm{~g} / \mathrm{kg}$ of ethanol or vehicle. Gel densitometric analysis has been carried out by using the public domain 'Image J' software (http://imagej.nih.gov/ij/). The vertical lines in the figure indicate pooled standard error means (SEM) derived from appropriate error mean square in the ANOVA. Asterisks indicate significant differences between groups $(* P<0.05)$

(Raivio, Miettinen \& Kiianmaa 2014) and chronic EtOH vapor-induced PAE was shown to increase the expression of BDNF in the ventral tegmental area of the offspring (Finegersh \& Homanics 2014). Our results appear to support an increase in BDNF expression in the frontal cortex of PAE offsprings, while EtOH administration reduced the BDNF expression to levels comparable to those showed by the non-PAE male offspring. 
(a)

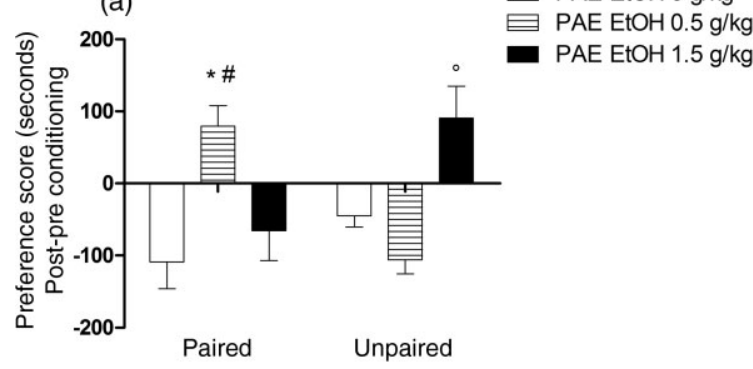

(b)

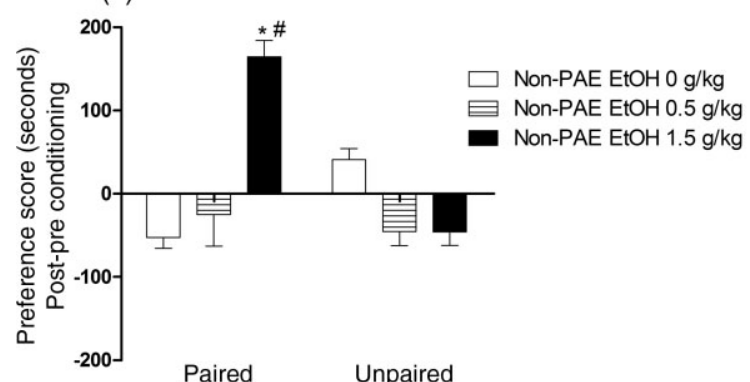

Figure $4 \mathrm{EtOH}$-induced conditioned place preference (CPP) is differently modulated in PAE (a) and non-PAE (b) male offspring. (a) PAE offspring showed a significant increase in preference scores (seconds) for the $\mathrm{EtOH}$-paired side $(0.5 \mathrm{~g} / \mathrm{kg})$ while a significant increase in preference scores (seconds) for the $\mathrm{EtOH}$-unpaired side $(1.5 \mathrm{~g} / \mathrm{kg}$ ). (b) Non-PAE offspring showed a significant increase in preference scores (seconds) for the EtOH-paired side $(1.5 \mathrm{~g} / \mathrm{kg}$ ) but no $\mathrm{EtOH}$-induced preference $(0.5 \mathrm{~g} / \mathrm{kg})$. Data are presented as mean $\pm \mathrm{SEM}$. $* \mathrm{EtOH} 0 \mathrm{~g} / \mathrm{kg}$ versus $\mathrm{EtOH} 0.5 \mathrm{~g} / \mathrm{kg}$ (a, paired side); *EtOH $0 \mathrm{~g} / \mathrm{kg}$ versus EtOH $1.5 \mathrm{~g} / \mathrm{kg}$ (b, paired side); \#EtOH $0.5 \mathrm{~g} / \mathrm{kg}$ versus $\mathrm{EtOH} 1.5 \mathrm{~g} / \mathrm{kg}$ (a and b, paired side); ${ }^{\circ} \mathrm{EtOH} 0 \mathrm{~g} / \mathrm{kg}$ versus $\mathrm{EtOH} 0.5 \mathrm{~g} / \mathrm{kg}$ or $1.5 \mathrm{~g} / \mathrm{kg}$ (a, unpaired side). Tukey's post hoc test $(P<0.05)$

Although the impact of paternal alcoholism may not be as great as that associated with maternal alcoholism, our data strongly suggest that it should not be undervalued, thus indicating that paternal alcohol consumption produced key and permanent changes in the offspring's brain. Several studies support the view that paternal consumption of alcohol is associated with developmental abnormalities. PAE seems to be associated with significant changes in infant birth weight and ventricular septal defects (Little \& Sing 1987; Tarter, Jacob \& Bremer 1989; Passaro et al. 1998). Moreover, it has been shown that chronic paternal alcohol abuse causes cognitive impairment and hyperactivity, even in children raised by nondrinker adoptive fathers (Hegedus, Alterman \& Tarter 1984; Tarter et al. 1989). Some reports from rodent studies evidenced that PAE may elicit developmental abnormalities, as changes in organ weights including the brain (Tanaka, Suzuki \& Arima 1982; Abel 1993), thickening of cortical layers (Jamerson, Wulser \& Kimler 2004), and reduction in testosterone levels (Abel 1989) and also behavioral abnormalities as decreased displacement behavior in response to novel environments or stressful events (Abel 1991), altered spatial learning performances (Wozniak et al. 1991), and altered immobility in a forced swim test (Abel \& Bilitzke 1990). Notably, such alterations did not result to be associated with father's stress and/or malnutrition due to PAE. However, other studies found no decreases in implantation sites or fetal weight, and no increases in prenatal mortality (Randall et al. 1982; Passaro et al. 1998) or soft tissue malformations (Randall et al. 1982) or any effect on brain or placental weight (Cake \& Lenzer 1985).

In contrast to the paucity of data on PAE and neurotrophins, the effects of maternal alcohol consumption during pregnancy on neurotrophins have received much greater attention and generated multiple lines of evidence. Findings from different laboratories have shown changes in NGF and BDNF and their receptors in most of the brain areas including cortex, hippocampus, striatum, hypothalamus, olfactory lobes and cerebellum and in target organs of EtOH intoxication (e.g. liver), regardless of the route of EtOH administration during pregnancy (vapor; parenteral administration; drinking water). These NGF/BDNF changes were also associated with behavioral alterations in offspring, such as in memory and learning abilities and sensitivity to EtOH consumption (Naassila et al. 2003; Aloe 2006; Hellemans et al. 2008, 2009; Fiore et al. 2009b; Ceccanti et al. 2012, 2013). Quite interestingly, the changes in neurotrophins and related behavioral parameters observed in PAE animal models and in other FASD animal models appear to be comparable, leading to the possibility that ethanol may disrupt brain cells directly, throughout its metabolites, by the way of ethanol-induced oxidative stress and/or genetic-epigenetic pathways.

Neurotrophins can modulate neuroadaptations in addictive behavior and alcohol consumption is influenced by the interaction between BDNF and dopamine (DA) transmission (Bosse \& Mathews 2011). It has been shown that EtOH exposure in sire mice may induce ADHD-like (attention deficit hyperactivity disorder) effects such as hyperactive, inattentive and impulsive behaviors in the offspring (Kim et al. 2014). Alterations of the DA signaling and DAT are commonly associated with ADHD, and an increased methylation of a $\mathrm{CpG}$ region of the DAT gene promoter in PAE animals is reported as potential epigenetic signature underlying the decrease of DAT protein and mRNA expression in cerebral cortex and striatum of PAE offspring (Kim et al. 2014).

In our hands, animals were sacrificed 1 week after place conditioning and, nevertheless, neurotrophins and DAT levels were shown to be affected by EtOH treatment. The rate at which DAT removes DA from the synapse can have a profound effect on DA signaling. This is best evi- 
denced by the severe cognitive deficits and hyperactivity of mice with no DA transporters (Van der Kooij \& Glennon 2007). We found a potential inverse relationship between EtOH doses and levels of DAT expression in olfactory lobes of male offspring from PAE mice (Fig. 3c). Thus, DAT expression was increased in animals that received $0.5 \mathrm{~g} / \mathrm{kg}$ EtOH, although the magnitude of DAT expression did not differ from that showed by offspring from non-PAE mice treated with the same EtOH dose. Despite the lack of difference between DAT expressions in offspring from PAE or non-PAE animals, these effects appear the consequence of the tendency toward an increase in DAT expression in offspring from PAE mice that did not receive any EtOH administration. DAT function is implicated in a number of DA-related disorders, including clinical depression and alcoholism (Getachew et al. 2010). DA neurotransmission plays an important role in EtOH consumption and alcohol-seeking behavior (for review, see Söderpalm \& Ericson 2013). EtOH intake potentiates DA release from ventral tegmental area dopaminergic (DAergic) neurons and increases DA extracellular levels in the nucleus accumbens (Koob 2014). Moreover, DAergic agents and lesion of the DA system modify EtOH self-administration, thus confirming a specific role for DA in EtOH reward (Di Chiara 1997). Olfactory lobes are a key limbic structure and the greatest sensitivity to variations in the total amount of DAT protein was observed with the same low dose eliciting the EtOH-induced rewarding effects. Consequently, these data support the view that $\mathrm{EtOH}$ reward and $\mathrm{EtOH}-$ seeking behavior of PAE male offspring may be positively associated with the amount of DAT protein and, likely, with reduced DA in the olfactory bulb. In this regard, the reduction in DA levels is reminding of the withdrawal syndrome hypothesis and withdrawal-induced decrease in DA transmission, as the neural correlate of increasing motivation to EtOH-seeking behavior (Koob 2014). Hence, adult male offsprings from PAE seem to exhibit some of the neuroadaptations (i.e. changes in NGF, BDNF and DA signaling) known to produce changes in sensitivity to EtOH-mediated effects (withdrawal symptoms) following chronic exposure and discontinuation to alcohol intake.

As previously observed, the findings of the present study indicate that alcohol drinking behavior may be epigenetically transferred through the father lineage. Our working hypothesis posits that alcohol is an epimutagen determining specific long-lasting changes affecting the next generations and their alcohol abuse, although the ethanol mechanism of action as epimutagen is not fully known at least in PAE. However, several FASD studies indicate that this spectrum of disorders due to prenatal ethanol arises from a complex interplay of genetic and epigenetic factors (reviewed in Mead \& Sarkar 2014). The present results demonstrate a direct impact of PAE on basal NGF and BDNF levels in target tissues of EtOH intoxication and the lowering of the threshold to EtOHelicited rewarding effects in male offspring although a limitation of the present study may be represented by the small number of experimental subjects used in the testings (as in any animal model study) that could have in some way affected the results.

In conclusion, since EtOH consumption before and at the time of procreation is a key factor in healthy child development, the present study may represent a step forward in the attempt to disclose some biomolecular processes and behavioral responses to rewarding outcomes underlying the transgenerational effects of alcohol intoxication. Therefore, these results may be of interest for the investigation of human addiction and in particular for those interested in the epigenetic programming via the paternal lineage.

\section{Acknowledgements}

We thank Sara De Nicolo for her valuable contribution in managing PAE mice. This work was supported by CNR, Regione Lazio and CRARL Lazio. The funders had no role in study design, data collection and analysis, decision to publish or preparation of the manuscript.

\section{Disclosure/Conflict of Interest}

The authors declare no conflict of interest.

\section{Authors Contribution}

MauC and MF were responsible for the study concept and design. RC, VC, SC, GF, GG, PT, CC and MF contributed to the acquisition of animal data. VC, SC, GF, PT, CC, EP, RM and MarC performed the biomolecular analyses. MauC, $\mathrm{RC}, \mathrm{VC}$ and MF contributed to data analysis and interpretation of findings. RC, VC and MF drafted the manuscript. GC provided critical revision of the manuscript for important intellectual content. All authors critically reviewed content and approved final version for publication.

\section{References}

Abel E (2004) Paternal contribution to fetal alcohol syndrome. Addict Biol 9:127-133, discussion 135-6.

Abel EL (1989) Paternal and maternal alcohol consumption: effects on offspring in two strains of rats. Alcohol Clin Exp Res 13:533-541.

Abel EL (1991) Paternal alcohol consumption affects grooming response in rat offspring. Alcohol Fayettev N 8:21-23.

Abel EL (1993) Rat offspring sired by males treated with alcohol. Alcohol Fayettev N 10:237-242.

Abel EL (1999) What really causes FAS? Teratology 59:4-6.

Abel EL (2009) Fetal alcohol syndrome: same old, same old. Addiction 104:1274-1275, discussion 1279-80. 
Abel EL, Bilitzke P (1990) Paternal alcohol exposure: paradoxical effect in mice and rats. Psychopharmacology (Berl) 100:159164.

Abel EL, Dintcheff BA (1986) Effects of prenatal alcohol exposure on behavior of aged rats. Drug Alcohol Depend 16:321330.

Allen SJ, Dawbarn D (2006) Clinical relevance of the neurotrophins and their receptors. Clin Sci 110:175-191.

Alleva E, Aloe L, Bigi S (1993) An updated role for nerve growth factor in neurobehavioural regulation of adult vertebrates. Rev Neurosci 4:41-62.

Aloe L (2001) Nerve growth factor and neuroimmune responses: basic and clinical observations. Arch Physiol Biochem 109:354-356.

Aloe L (2006) Alcohol intake during prenatal life affects neuroimmune mediators and brain neurogenesis. Ann Ist Super Sanita 42:17-21.

Aloe L, Alleva E, Fiore M (2002) Stress and nerve growth factor: findings in animal models and humans. Pharmacol Biochem Behav 73:159-166.

Aloe L, Bracci-Laudiero L, Bonini S, Manni L (1997) The expanding role of nerve growth factor: from neurotrophic activity to immunologic diseases. Allergy 52:883-894.

Aloe L, Bracci-Laudiero L, Tirassa P (1993) The effect of chronic ethanol intake on brain NGF level and on NGF-target tissues of adult mice. Drug Alcohol Depend 31:159-167.

Aloe L, Tirassa P (1992) The effect of long-term alcohol intake on brain NGF-target cells of aged rats. Alcohol 9:299-304.

Astley S (2004) Diagnostic Guide for Fetal Alcohol Spectrum Disorders: The 4-Digit Diagnostic Code, Third-2004, Seattle, WA: FAS Diagnostic and Prevention Network University of Washington.

Astley SJ (2013) Validation of the fetal alcohol spectrum disorder (FASD) 4-Digit Diagnostic Code. J Popul Ther Clin Pharmacol J Thérapeutique Popul Pharamcologie Clin 20:e416-e467.

Bakoyiannis I, Gkioka E, Pergialiotis V, Mastroleon I, Prodromidou A, Vlachos GD, Perrea D (2014) Fetal alcohol spectrum disorders and cognitive functions of young children. Rev Neurosci 25:631-639.

Bekinschtein P, Cammarota M, Medina JH (2014) BDNF and memory processing. Neuropharmacology 76 (Pt C):677-683.

Bielawski DM, Abel EL (1997) Acute treatment of paternal alcohol exposure produces malformations in offspring. Alcohol Fayettev N 14:397-401.

Bonini S, Lambiase A, Lapucci G, Properzi F, Bresciani M, Bracci Laudiero ML, Mancini MJ, Procoli A, Micera A, Sacerdoti G, Bonini S, Levi-Schaffer F, Rasi G, Aloe L (2002) Nerve growth factor and asthma. Allergy 57 (Suppl. 72):1315.

Bosse KE, Mathews TA (2011) Ethanol-induced increases in extracellular dopamine are blunted in brain-derived neurotrophic factor heterozygous mice. Neurosci Lett 489:172-176.

Cake H, Lenzer I (1985) On effects of paternal ethanol treatment on fetal outcome. Psychol Rep 57:51-57.

Ceccanti M, De Nicolo S, Mancinelli R, Chaldakov G, Carito V, Ceccanti M, Laviola G, Tirassa P, Fiore M (2013) NGF and BDNF long-term variations in the thyroid, testis and adrenal glands of a mouse model of fetal alcohol spectrum disorders. Ann Ist Super Sanita 49:383-390.

Ceccanti M, Mancinelli R, Tirassa P, Laviola G, Rossi S, Romeo M, Fiore M (2012) Early exposure to ethanol or red wine and longlasting effects in aged mice. A study on nerve growth factor, brain-derived neurotrophic factor, hepatocyte growth factor, and vascular endothelial growth factor. Neurobiol Aging 33:359-367.

Chao MV (2000) Trophic factors: an evolutionary cul-de-sac or door into higher neuronal function? J Neurosci Res 59:353355.

Chao MV, Hempstead BL (1995) p75 and Trk: a two-receptor system. Trends Neurosci 18:321-326.

Chao MV, Rajagopal R, Lee FS (2006) Neurotrophin signalling in health and disease. Clin Sci 110:167-173.

Cirulli F, Adriani W, Laviola G (1997) Sexual segregation in infant mice: behavioural and neuroendocrine responses to d-amphetamine administration. Psychopharmacol Berl. 134:140-152.

Cohen S, Levi-Montalcini R, Hamburger V (1954) A nerve growth-stimulating factor isolated from sarcomas 37 and 180. Proc Natl Acad Sci U S A 40:1014-1018.

Cuello AC (1983) Brain Microdissection Techniques, Chichester: Wiley.

De Nicolo S, Tarani L, Ceccanti M, Maldini M, Natella F, Vania A, Chaldakov GN, Fiore M (2013) Effects of olive polyphenols administration on nerve growth factor and brain-derived neurotrophic factor in the mouse brain. Nutrition 29:681687.

Di Chiara G (1997) Alcohol and dopamine. Alcohol Health Res World 21:108-114.

Finegersh A, Homanics GE (2014) Paternal alcohol exposure reduces alcohol drinking and increases behavioral sensitivity to alcohol selectively in male offspring. PLoS ONE 9:e99078.

Fiore M, Chaldakov GN, Aloe L (2009a) Nerve growth factor as a signaling molecule for nerve cells and also for the neuroendocrine-immune systems. Rev Neurosci 20:133-145.

Fiore M, Laviola G, Aloe L, di Fausto V, Mancinelli R, Ceccanti M (2009b) Early exposure to ethanol but not red wine at the same alcohol concentration induces behavioral and brain neurotrophin alterations in young and adult mice. Neurotoxicology 30:59-71.

Fiore M, Mancinelli R, Aloe L, Laviola G, Sornelli F, Vitali M, Ceccanti M (2009c) Hepatocyte growth factor, vascular endothelial growth factor, glial cell-derived neurotrophic factor and nerve growth factor are differentially affected by early chronic ethanol or red wine intake. Toxicol Lett 188:208-213.

Freund-Michel V, Frossard N (2008) The nerve growth factor and its receptors in airway inflammatory diseases. Pharmacol Ther 117:52-76.

Getachew B, Hauser SR, Taylor RE, Tizabi Y (2010) Alcoholinduced depressive-like behavior is associated with cortical norepinephrine reduction. Pharmacol Biochem Behav 96:395-401.

Goodlett CR, Gilliam DM, Nichols JM, West JR (1989) Genetic influences on brain growth restriction induced by development exposure to alcohol. Neurotoxicology 10:321-334.

Goodlett CR, Horn KH (2001) Mechanisms of alcohol-induced damage to the developing nervous system. Alcohol Res Health 25:175-184.

He Y-Y, Zhang X-Y, Yung W-H, Zhu J-N, Wang J-J (2013) Role of BDNF in central motor structures and motor diseases. Mol Neurobiol 48:783-793.

Hegedus AM, Alterman AI, Tarter RE (1984) Learning achievement in sons of alcoholics. Alcohol Clin Exp Res 8:330-333.

Hellemans KG, Sliwowska JH, Verma P, Weinberg J (2009) Prenatal alcohol exposure: fetal programming and later life vulnerability to stress, depression and anxiety disorders. Neurosci Biobehav Rev 34:791-807. 
Hellemans KG, Verma P, Yoon E, Yu W, Weinberg J (2008) Prenatal alcohol exposure increases vulnerability to stress and anxiety-like disorders in adulthood. Ann $\mathrm{N}$ Acad Sci 1144:154-175.

Hempstead BL (2014) Deciphering proneurotrophin actions. Handb Exp Pharmacol 220:17-32.

Huang EJ, Reichardt LF (2003) Trk receptors: roles in neuronal signal transduction. Annu Rev Biochem 72:609-642.

Jamerson PA, Wulser MJ, Kimler BF (2004) Neurobehavioral effects in rat pups whose sires were exposed to alcohol. Brain Res Dev Brain Res 149:103-111.

Jones KL (1975) The fetal alcohol syndrome. Addict Dis 2:7988.

Kim P, Choi CS, Park JH, Joo SH, Kim SY, Ko HM, Kim KC, Jeon SJ, Park SH, Han SH, Ryu JH, Cheong JH, Han JY, Ko KN, Shin CY (2014) Chronic exposure to ethanol of male mice before mating produces attention deficit hyperactivity disorder-like phenotype along with epigenetic dysregulation of dopamine transporter expression in mouse offspring. J Neurosci Res 92:658-670.

Koob GF (2014) Neurocircuitry of alcohol addiction: synthesis from animal models. Handb Clin Neurol. 125:33-54.

Levi-Montalcini R (1987) The nerve growth factor 35 years later. Science 237:1154-1162.

Little RE, Sing CF (1987) Father's drinking and infant birth weight: report of an association. Teratology 36:59-65.

Macchia T, Mancinelli R, Gentili S, Lugaresi EC, Raponi A, Taggi F (1995) Ethanol in biological fluids: headspace GC measurement. J Anal Toxicol 19:241-246.

Mead EA, Sarkar DK (2014) Fetal alcohol spectrum disorders and their transmission through genetic and epigenetic mechanisms. Front Genet 5:154.

Naassila M, Pierrefiche O, Beauge FJ, Sebire N, Daoust M (2003) Chronic ethanol exposure differentially regulates NOS1 mRNA levels depending on rat brain area. Neurosci Lett 338:221-224.

O'Malley KD, Nanson J (2002) Clinical implications of a link between fetal alcohol spectrum disorder and attention-deficit hyperactivity disorder. Can J Psychiatry 47:349-354.

Passaro KT, Little RE, Savitz DA, Noss J (1998) Effect of paternal alcohol consumption before conception on infant birth weight. ALSPAC Study Team. Avon Longitudinal Study of Pregnancy and Childhood. Teratology 57:294-301.

Raivio N, Miettinen P, Kiianmaa K (2014) Innate BDNF expression is associated with ethanol intake in alcohol-preferring
AA and alcohol-avoiding ANA rats. Brain Res 1579: 74-83.

Randall CL, Burling TA, Lochry EA, Sutker PB (1982) The effect of paternal alcohol consumption on fetal development in mice. Drug Alcohol Depend 9:89-95.

Servais L, Hourez R, Bearzatto B, Gall D, Schiffmann SN, Cheron G (2007) Purkinje cell dysfunction and alteration of longterm synaptic plasticity in fetal alcohol syndrome. Proc Natl Acad Sci USA 104:9858-9863.

Sofroniew MV, Howe CL, Mobley WC (2001) Nerve growth factor signaling, neuroprotection, and neural repair. Annu Rev Neurosci 24:1217-1281.

Söderpalm B, Ericson M (2013) Neurocircuitry involved in the development of alcohol addiction: the dopamine system and its access points. Curr Top Behav Neurosci. 13:127-161.

Streissguth AP, O'Malley K (2000) Neuropsychiatric implications and long-term consequences of fetal alcohol spectrum disorders. Semin Clin Neuropsychiatry 5:177-190.

Tabakoff B, Hoffman PL (2013) The neurobiology of alcohol consumption and alcoholism: an integrative history. Pharmacol Biochem Behav 113:20-37.

Tanaka H, Suzuki N, Arima M (1982) Experimental studies on the influence of male alcoholism on fetal development. Brain Dev 4:1-6.

Tarter RE, Jacob T, Bremer DL (1989) Specific cognitive impairment in sons of early onset alcoholics. Alcohol Clin Exp Res 13:786-789.

Tsai G, Gastfriend DR, Coyle JT (1995) The glutamatergic basis of human alcoholism. Am J Psychiatry 152:332-340.

Van der Kooij MA, Glennon JC (2007) Animal models concerning the role of dopamine in attention-deficit hyperactivity disorder. Neurosci Biobehav Rev 31:597-618.

Ventura R, Coccurello R, Andolina D, Latagliata EC, Zanettini C, Lampis V, Battaglia M, D’Amato FR, Moles A (2013) Postnatal aversive experience impairs sensitivity to natural rewards and increases susceptibility to negative events in adult life. Cereb Cortex 23:1606-1617.

Wozniak DF, Cicero TJ, Kettinger L, Meyer ER (1991) Paternal alcohol consumption in the rat impairs spatial learning performance in male offspring. Psychopharmacology (Berl) 105:289-302.

Yanev S, Aloe L, Fiore F, Chaldakov GN (2013) Neurotrophic and metabotrophic potential of nerve growth factor and brain-derived neurotrophic factor: linking cardiometabolic and neuropsychiatric diseases. World J Pharmacol 2:92-99. 